\title{
Wound healing in postbariatric body contouring surgery
}

\author{
Olimpiu Bota, Martin Schreiber, Florian Bönke, Dominika Teather, Adrian Dragu \\ Department of Plastic and Hand Surgery, University Center of Orthopedics and Trauma Surgery, University Hospital Carl Gustav Carus, \\ Dresden 01307, Germany.
}

Correspondence to: Prof. Adrian Dragu, Department of Plastic and Hand Surgery, University Center of Orthopedics and Trauma Surgery, University Hospital Carl Gustav Carus, Fetscherstraße 74, Dresden 01307, Germany. E-mail: adrian.dragu@uniklinikum-dresden.de

How to cite this article: Bota O, Schreiber M, Bönke F, Teather D, Dragu A. Wound healing in postbariatric body contouring surgery. Plast Aesthet Res 2018;5:30. http://dx.doi.org/10.20517/2347-9264.2018.47

Received: 12 Jun 2018 Accepted: 24 Jul 2018 Published: 23 Aug 2018

Science Editor: Raymund Engelbert Horch Copy Editor: Jun-Yao Li Production Editor: Huan-Liang Wu

\begin{abstract}
Due to the continuous development in the field of bariatric surgery, there is an increasing need for postbariatric body contouring surgery. The morbidity of postbariatric patients predisposes them to develop wound healing complications. In this article we describe the preoperative, intraoperative and surgical factors influencing the wound healing and therefore the final outcome. The most common postbariatric body contouring procedures, including brachioplasty, breast contouring surgery, abdominoplasty/circumferential body lift and medial thigh lift are being discussed in terms of wound healing characteristics and subsequent complications. The preoperative preparing as well as special operative techniques are described in order to achieve a low rate of wound healing complications.
\end{abstract}

Keywords: Body contouring, postbariatric surgery, wound healing

\section{INTRODUCTION}

According to the World Health Organization (WHO) in 2016, 39\% of the world population was overweight and $13 \%$ of this group were obese. Overweight is defined by a body mass index (BMI, $\mathrm{kg} / \mathrm{m}^{2}$ ) $\geq 25$ and obesity is defined by a $\mathrm{BMI} \geq 30^{[1]}$. In order to reduce the comorbidities associated with overweight like diabetes mellitus type 2 and certain types of cancer and cardiovascular diseases ${ }^{[2]}$, the current guidelines for obesity recommend a weight loss of at least five percent body weight in patients with a BMI $\leq 35$ and a weight loss of at least ten percent body weight in patients with a $\mathrm{BMI} \leq 35$. The therapeutical change of life style, diet re- 
strictions and physical activity as well as pharmacotherapy are the first choices in reducing the body weight. In advanced cases the bariatric surgery comes into play, especially the laparoscopic procedures being able to provide weight loss with reduced complication rates ${ }^{[3]}$. According to the German guideline for Adiposity and Metabolic Disease Surgery, 2018, the surgical therapy is recommended in patients with a BMI $\geq 40$, in patients with a BMI between 35 and 40 and comorbidities associated with obesity and in patients with a BMI between 30 and 35 and type 2 diabetes mellitus ${ }^{[4]}$. In 2013 there were about half million bariatric surgical interventions ${ }^{[3]}$ performed worldwide, which implements the need for these procedures.

After a long process of reducing the BMI and achieving the ideal body weight, patients still have to face the next challenge. Regardless of the methods used to successfully reduce the weight, massive weight loss patients often develop redundant, hanging skin and fat depots which cannot be removed by diet, physical exercise or medication. Functional handicaps, rashes, skin infections, difficult body hygiene, self-confidence or daily problems finding appropriate clothing motivate the patient to go to the plastic surgeon. Although the successful weight loss reduces some of the medical risks, the psychosocial and functional problems often remain a problem for these patients ${ }^{[5]}$. Up to $89 \%$ of the postbariatric patients complain of problems with the redundant skin and up to half of these patients find this condition to be worse than the initial obesity ${ }^{[6]}$. In the era of bariatric surgery, the body contouring surgery (BCS) plays a key role in achieving the final result for the obese patients.

The postbariatric BCS uses reconstructive procedures to improve the physical and psychological status of the patient and by having a medical indication, it distinguishes itself from the sheer aesthetic interventions. Up to $74 \%$ of patients who underwent bariatric surgery opt for a body contouring procedure ${ }^{[7]}$. Although it has a medical indication, the health insurances only cover a fraction of the treatment of these patients and therefore the demand for postbariatric body contouring remains higher than the actual performed interventions. In a large populational study, Lazzati et al ${ }^{[8]}$ found that only $21 \%$ of bariatric patients undergo BCS.

Several studies have proven that BCS in postbariatric patients is prone to more complications than in patients who did not receive weight loss surgery ${ }^{[9,10]}$. The overall early complication rate in the literature varies from $45 \%$ to $70 \%$ and includes hematoma, seroma, wound dehiscence, infection, deep vein thrombosis and pulmonary embolism, whereas wound healing disorders and seroma formation appear to be by far the most common ${ }^{[10,11]}$. Several factors seem to be involved in the development of these wound complications, including preoperative factors, intraoperative factors and surgical procedure factors.

\section{PREOPERATIVE FACTORS}

Patients losing weight after a bariatric procedure are known to have nutritional deficiencies. Patients undergoing postbariatric body contouring have been proven to have low prealbumin and hemoglobin, vitamin $\mathrm{A}, \mathrm{C}$, $\mathrm{B}$ complex, iron, zinc and selenium deficiencies as well hyperhomocystinemia ${ }^{[12,13]}$. These factors are known to be essential for wound healing and therefore leave the postbariatric patient at risk for wound healing disorders. There is evidence that the perioperative nutritional supplementation can improve the wound healing process in these patients and decrease the complication rate. Some authors even recommend an extended nutritional evaluation before beginning the body contouring procedures ${ }^{[14]}$.

One of the most significant risk factors for the BCS appears to be the BMI at the time of surgery. In spite of weight loss surgery, physical exercise and strict diets, the BMI in some patients reaches a stationary level, which cannot be improved anymore. The existence of large abdominal aprons, excessive medial thigh skin and fatty tissue as well as macromastia prevent the patient from performing daily activities and exercise and result in a vicious circle, where further weight loss is not possible. Most studies have proven a high BMI to be an important risk factor for developing complications after $\mathrm{BCS}^{[10]}$. As a matter of consequence, the amount of tissue removed is also correlated with increased wound complications ${ }^{[14]}$. The combination of large opera- 
tive wounds and poorly vascularized, excessive fatty tissue will most probably lead to fat necrosis, development of seromas and cellulitis as well as wound dehiscence. Certain operative techniques have been developed in order to approach this problem.

Smoking can also have an important impact on wound healing. Some studies describe that in smokers the relative risks of developing a wound infection after contouring breast surgery are 3.8 times higher and after medial thigh lift 7.74 times higher respectively ${ }^{[15,16]}$. These studies suggest that patients should be advised to quit smoking six weeks before the BCS.

The season in which the BCS takes place can also be an influential factor on wound healing. In a study on 602 patients after postbariatric BCS, Duscher et al. ${ }^{[17]}$ found a statistically significant difference in the development of wound infections between patients operated in the warm seasons compared to those operated in the cold seasons $(10.29 \%$ vs. $4.08 \%, P=0.0071)$. Although the frequency of BCS appears to be higher in the cold seasons, the patients operated in spring and summer seem to develop more wound complications. This could be attributed to a higher bacterial load at the surgical site as well as to deficient hygiene.

\section{INTRAOPERATIVE FACTORS}

The intraoperative administration of higher volumes of fluids has been associated with the formation of seroma, hematoma and wound healing problems ${ }^{[18]}$. Longer periods of oxygen desaturation $(\leq 92 \%)$ have also been associated with wound complications, while longer periods of low $\left(35.6^{\circ}\right)$ intraoperative core temperature, seem to increase the rate of hematomas and bleeding ${ }^{[19]}$. Transfusion of blood products has also been associated with the development of wound complications ${ }^{[20]}$.

Keeping a warm environment during surgery and a constant core temperature of $37^{\circ}$, accurate surgery without bleeding and precise administration of fluids during surgery can significantly influence the outcome of the operation and favour the uncomplicated, primary wound healing.

\section{SURGICAL FACTORS}

Brachioplasty, breast reduction/mastopexy, abdominoplasty/circumferential body lift and thigh lift are the most common postbariatric $\mathrm{BCS}^{[7,8]}$. Although the general patient features are the same, each body region and intervention has different characteristics and different rates of wound complications. In our practice we avoid addressing more than one body region per procedure. Studies have shown that multi-stage procedures reduce the risk of postoperative complications in comparison to long one-stage procedures. Especially, in the context of "pay for performance" probably future reimbursement from the health insurance will be reduced in treated cases with high revision operations due to postoperative complications in single-step procedures.

In the following we will address each procedure separately, analyzing the probability of wound complications and the techniques to avoid them.

Brachioplasty is one of the least frequent postbariatric BCS and has a rather low rate of postoperative complications compared to other forms of BCS. In a review on 1065 brachioplasties, there were $29.8 \%$ complications, whereby hematoma and seroma were found to be the most common early postoperative complication and poor scarring the most common late complication ${ }^{[21,22]}$. In our experience, the use of liposuction in the same procedure before performing the skin resection ensures a safe layer of dissection, without jeopardizing the superficial and deep veins as well as the lymph collectors, while ensuring the best aesthetical result [Figures 1-4]. The approach of the axillary fold is best done by a M-Y axilloplasty, which ensures an optimal aesthetic result while avoiding the postoperative wound complications and scar contractures ${ }^{[23]}$. 


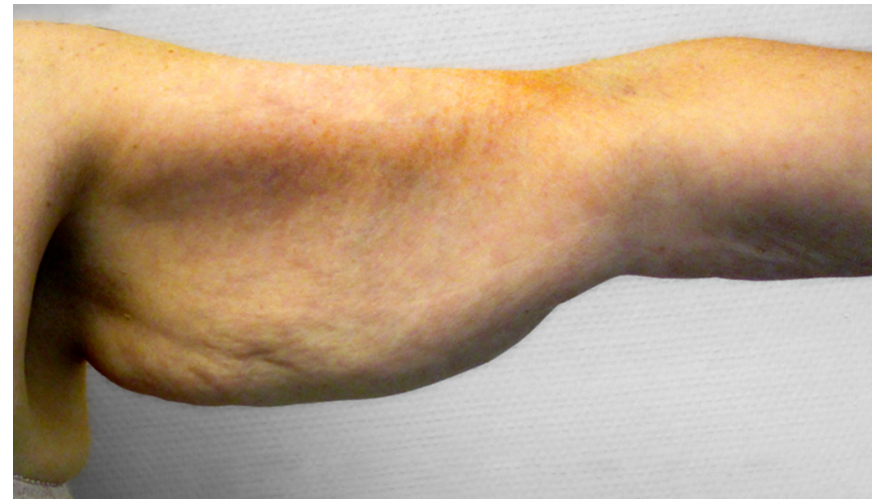

Figure 1. Redundant arm skin after bariatric surgery

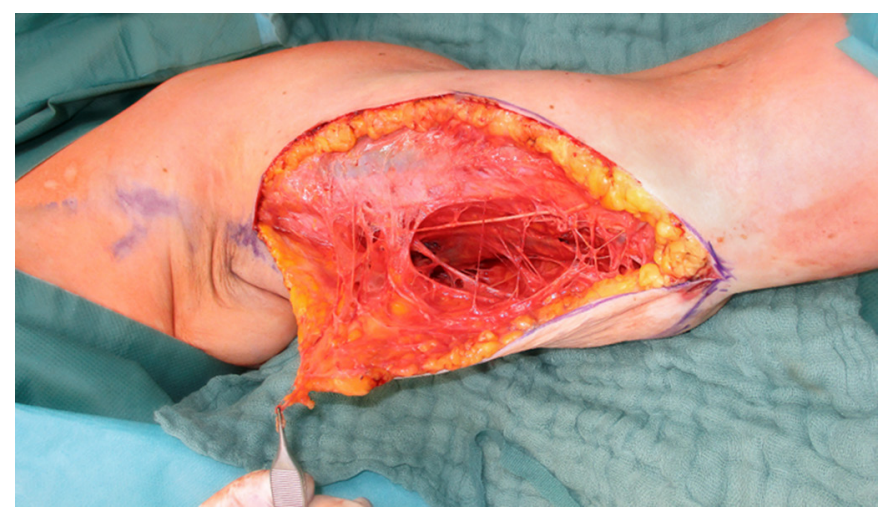

Figure 2. Medial upper arm region after liposuction prior to resection. The superficial fat layer as well as the blood vessels, nerves and lymph collectors are preserved

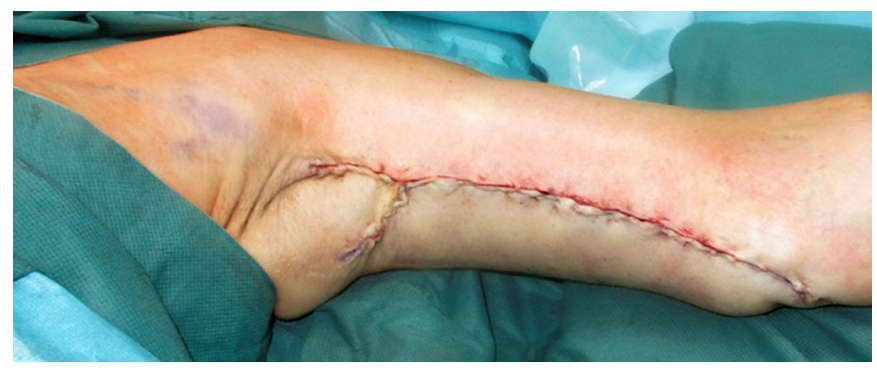

Figure 3. Postoperative result with simultaneous liposuction and M-Y axilloplasty

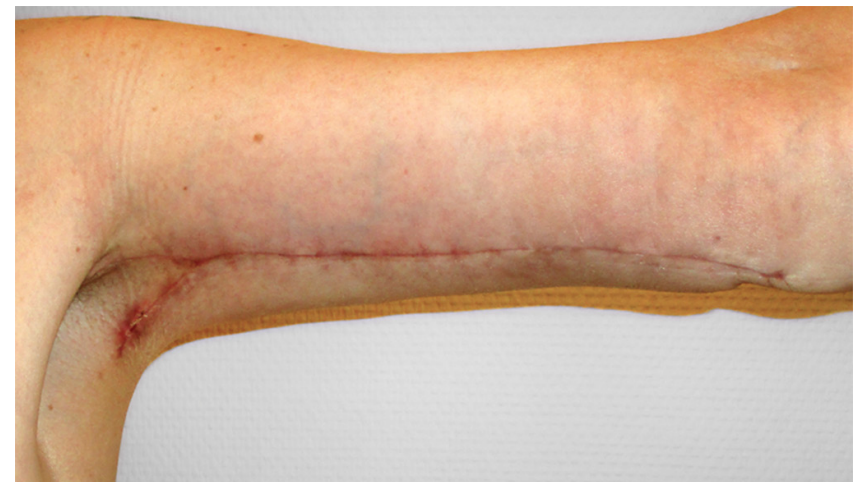

Figure 4. Result 6 weeks postoperative after brachioplasty with simultaneous liposuction and M-Y axilloplasty 
Breast contouring surgery after massive weight loss encompasses a wide spectrum of operations, including breast reduction techniques, mastopexy as well as autologous and implant augmentation. Most of these techniques use the Wise pattern skin incision, which results in an inversed-T scar. The majority of the postoperative wound complications appear in the inverted T-junction area [Figure 5], resulting from poor vascularization of the two pillars and excessive suture tension. Fatty tissue necrosis from rearrangement of tissue can also appear, causing delayed wound healing, infection and increased surgical revision rates. The nippleareola complex (NAC) represents a special issue in breast contouring. Breasts after massive weight loss are characterized by advanced ptosis and subsequent extra-long NAC pedicles. This often causes deficient NAC vascularization followed necrosis and delayed wound healing. The overall complication rate for postbariatric breast contouring surgery is reported to be between $35.7 \%$ and $57 \%$, mostly consisting of surgical wound problems ${ }^{[15,20]}$. We prefer the use of autoaugmentation with a lateral and medial flap for breast reshaping, as this means killing two birds with one stone: achieving the breast augmentation without foreign matter while performing a lateral lift at the same time.

Abdominoplasty is the most frequent performed BCS. The presence of an abdominal apron represents the most common complaint of patients after massive weight loss. Skin macerations, intertrigo, rashes, difficult hygiene cause a great amount of discomfort for the patient, even more than before the weight loss. The addition of the hypertrophy of mons pubis renders this as a high symptomatic area which has to be addressed primarily, in order to improve the patient's life quality. These patients usually associate redundant fat tissue and skin in the epigastric, flank and back areas. The anterior abdominal wall can be addressed in one procedure, associating the abdominoplasty with a mons pubis lift. The epigastric and the flank regions can be approached either by a fleur-de-lis procedure or by performing a liposuction of the upper and lateral quadrants and the flanks before performing the paniculectomy, in a lipoabdominoplasty procedure. The fleur-de-lis procedure offers the largest amount of tissue excision in exchange for the longest scars. In this case the reduced vascularity of the skin flaps around the T-scar, the suture tension and the patient specific risk factors represent a hot spot for wound complications. The lipoabdominoplasty removes the excessive fat remaining in the abdominal wall while preserving the innervation and blood supply. The upper flap can be then caudally mobilized without extended undermining while preserving the vascularization of zones I and III while resecting zone II. The umbilicus can be repositioned by dissecting a narrow cranial tunnel and the skin can finally be tensioned and repositioned in order to achieve a tight, aesthetical result, while lifting the mons pubis at the same time. By maintaining the flap vascularity and removing the troublesome excessive fat tissue at the same time, this technique provides improved wound healing rates ${ }^{[24]}$. We prefer the lift of the lower back in a secondary procedure in a prone position, as the circumferential body lift provides enhanced wound complications while being economically disadvantageous ${ }^{[25]}$.

The incidence of postoperative complications after abdominoplasty is estimated around 57\%, mostly consisting of seromas, wound healing problems and hematomas. Avoiding these problems requires good patient selection and operative planning, the placement of the operative incision below the contaminated infraabdominal fold, preserving the scarpa fascia, using liposuction when possible, using progressive tension sutures to reduce the dead space and seroma formation ${ }^{[26,27]}$ and ensuring a tensionless wound closure.

The medial thigh lift may be considered the "problem child" of the postbariatric BCS. Although the operative indication is strongly motivated by the patient discomforts, including friction, rashes and difficulty with ambulation, this intervention is marked by the high rate of postoperative complications. Complication rates as high as $69 \%$ to $78 \%{ }^{[16,20]}$ have been reported. The troublesome anatomical area, with superficial veins and lymph collectors running in the subcutaneous tissue predispose the patient to development of seromas, lymphoceles [Figures 6 and 7], hematomas and delayed wound healing. The use of the horizontal inguinal excision with the resulting T-scar adds more risk for wound dehiscence in this area. The use of liposuction 


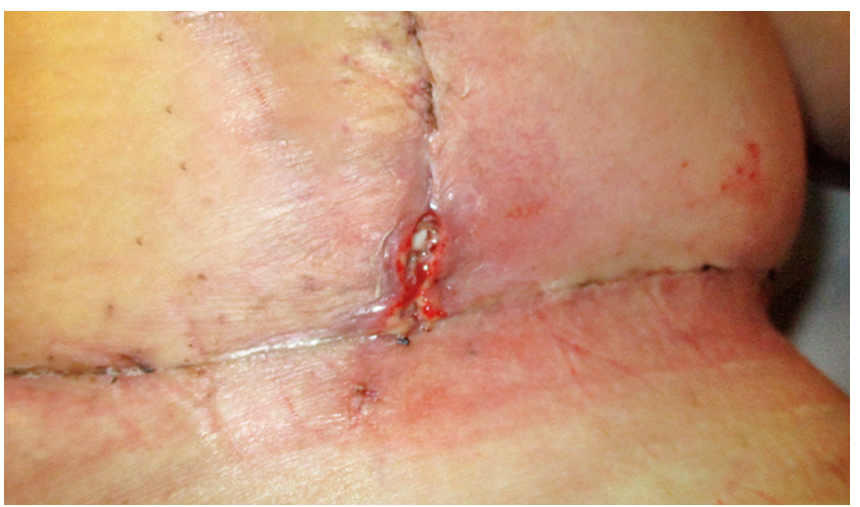

Figure 5. Wound dehiscence of the inverted T-scar after breast autoaugmentation with a lateral and medial flap

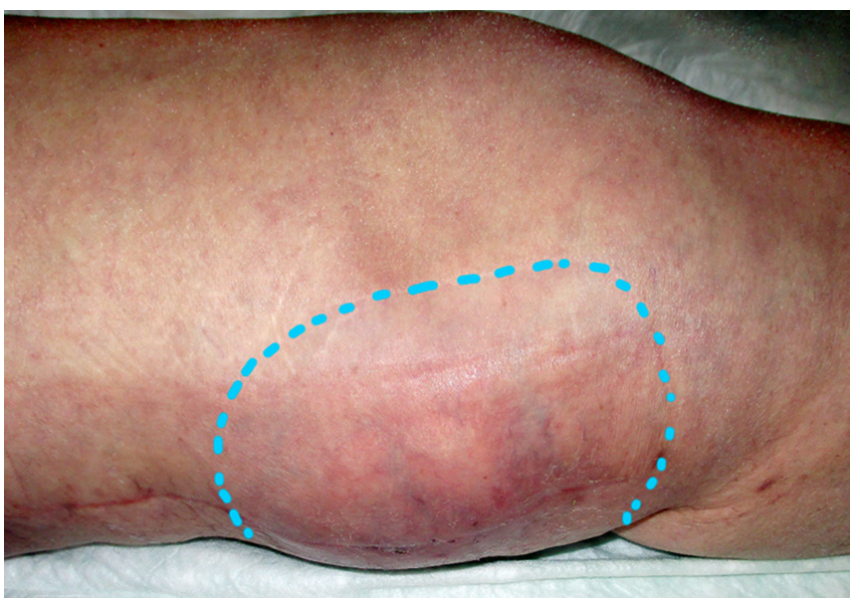

Figure 6. Postoperative lymphocele in the distal third of the scar after medial thigh lift on the left leg

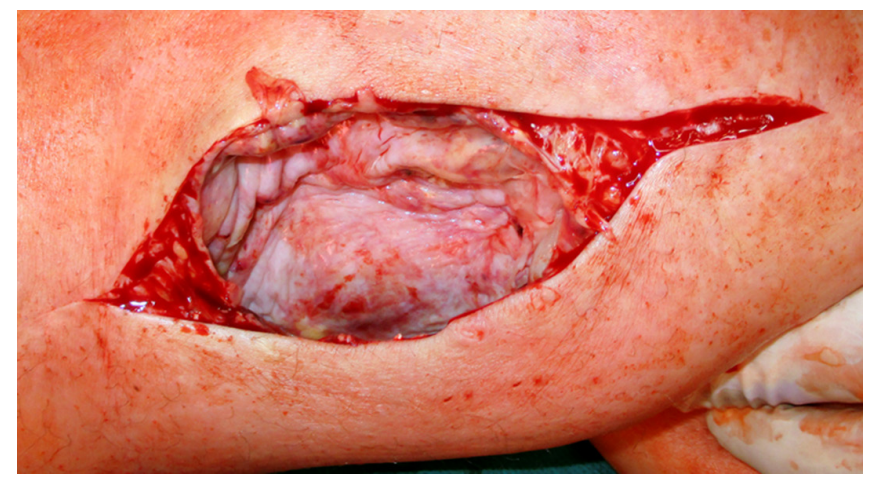

Figure 7. Postoperative lymphocele with typical pseudocapsule after medial thigh lift - intraoperative view before complete capsulectomy

in thigh lift is crucial in reducing the morbidity. Serial liposuctions before paniculectomy are prerequisite, in order to reduce the excessive fatty tissue remaining around the thigh. We find the use of a concomitant liposuction with the thigh lift to be of special importance. The liposuction provides a clear dissection plane, sparing the superficial veins and especially the lymph collectors lying above the deep fascia while reducing the residual fatty tissue and reducing complications ${ }^{[28]}$. 


\section{CONCLUSION}

Obesity is considered by many to be a world epidemic. With the continuous growth of bariatric surgery, the number of patients addressing the Plastic Surgeon after massive weight loss continues to grow. Although BCS provides a high improvement in quality of life, the incidence of postoperative complications and especially wound healing problems remains high. Patient selection, thorough preoperative preparation, atraumatic surgery with use of adjunctive techniques like liposuction and preservation of important anatomical structures can lead to improved wound healing rates and less complications. Further research needs to be done in order to reduce the large amount of dead space resulting after these operations and hence reduce the morbidity.

\section{DECLARATIONS}

Acknowledgments

Mr. Thomas Albrecht supported the processing of the figures.

\section{Authors' contributions}

Conception and design of the study, performed data collecting and analysis as well as manuscript editing: Bota O, Schreiber M, Bönke F, Teather D, Dragu A

\section{Availability of data and materials}

Not applicable.

\section{Financial support and sponsorship}

None.

\section{Conflicts of interest}

All authors declare that there are no conflicts of interest.

\section{Ethical approval and consent to participate}

Not applicable.

\section{Consent for publication}

Not applicable.

\section{Copyright}

(c) The Author(s) 2018.

\section{REFERENCES}

1. World Health Organisation. Obesity and overweight. Available from: URL: http://www.who.int/en/news-room/fact-sheets/detail/obesityand-overweight. [Last accessed on 7 Aug 2018]

2. González-Muniesa P, Mártinez-González MA, Hu FB, Després JP, Matsuzawa Y, Loos RJF, Moreno LA, Bray GA, Martinez JA. Obesity. Nat Rev Dis Primers 2017;3:17034.

3. Bray GA, Frühbeck G, Ryan DH, Wilding JPH. Management of obesity. Lancet 2016;387:1947-56.

4. AWMF online. S3-Leitlinie: Chirurgie der Adipositas und metabolischer Erkrankungen. Available from: http://www.awmf.org/uploads/ tx_szleitlinien/088-001m_S3_Chirurgie-Adipositas-metabolische-Erkrankugen_2018-02.pdf [Last accessed on 7 Aug 2018]

5. Giordano S, Victorzon M, Koskivuo I, Suominen E. Physical discomfort due to redundant skin in post-bariatric surgery patients. J Plast Reconstr Aesthet Surg 2013;66:950-5.

6. Herpertz S, Kielmann R, Wolf AM, Langkafel M, Senf W, Hebebrand J. Does obesity surgery improve psychosocial functioning? A systematic review. Int J Obes 2003;27:1300-14.

7. Kitzinger HB, Abayev S, Pittermann A, Karle B, Kubiena H, Bohdjalian A, Langer FB, Prager G, Frey M. The prevalence of body contouring surgery after gastric bypass surgery. Obes Surg 2012;22:8-12.

8. Lazzati A, Katsahian S, Maladry D, Gerard E, Gaucher S. Plastic surgery in bariatric patients: a nationwide study of 17,000 patients on 
the national administrative database. Surg Obes Relat Dis 2018;14:646-51.

9. Staalesen T, Olsén MF, Elander A. Complications of abdominoplasty after weight loss as a result of bariatric surgery or dieting/ postpregnancy. J Plast Surg Hand Surg 2012;46:416-20.

10. Kitzinger HB, Cakl T, Wenger R, Hacker S, Aszmann OC, Karle B. Prospective study on complications following a lower body lift after massive weight loss. J Plast Reconstr Aesthet Surg 2013;66:231-8.

11. Neaman KC, Hansen JE. Analysis of complications from abdominoplasty. Ann Plast Surg 2007;58:292-8.

12. Agha-Mohammadi S, Hurwitz DJ. Enhanced recovery after body-contouring surgery: reducing surgical complication rates by optimizing nutrition. Aesth Plast Surg 2010;34:617-25.

13. Agha-Mohammadi S, Hurwitz DJ. Potential impacts of nutritional deficiency of postbariatric patients on body contouring surgery. Plast Reconstr Surg 2008;122:1901-14.

14. Barbour JR, Iorio ML, Oh C, Tung TH, O’Neill PJ. Predictive value of nutritional markers for wound healing complications in bariatric patients undergoing panniculectomy. Ann Plast Surg 2015;75:435-8.

15. Gravante G, Araco A, Sorge R, Araco F, Delogu D, Cervelli V. Wound infections in body contouring mastopexy with breast reduction after laparoscopic adjustable gastric bandings: the role of smoking. Obes Surg 2008;18:721-7.

16. Poodt IGM, van Dijk MM, Klein S, Hoogbergen MM. Complications of lower body lift surgery in postbariatric patients. Plast Reconstr Surg Glob Open 2016;4:e1030.

17. Duscher D, Kiesl D, Aitzetmueller MM, Wenny R, Schableger K, Staud CJ, Pollhammer M, Shamiyeh A, Huemer GM. Seasonal impact on surgical site infections in body contouring surgery. Plast Reconstr Surg 2018; doi: 10.1097/PRS.0000000000004677.

18. Cohen B, Meilik B, Weiss-Meilik A, Tarrab A, Matot I. Intraoperative factors associated with postoperative complications in body contouring surgery. J Surg Res 2018;221:24-9.

19. Coon D, Michaels J, Gusenoff JA, Chong T, Purnell C, Rubin JP. Hypothermia and complications in postbariatric body contouring. Plast Reconstr Surg 2012;130:443-8.

20. García Botero A, García Wenninger M, Fernández Loaiza D. Complications after body contouring surgery in postbariatric patients. Ann Plast Surg 2017;79:293-7.

21. Miotto G, Ortiz-Pomales Y. Arm contouring: review and current concepts. Aesthet Surg J 2018;37:1.

22. Sisti A, Cuomo R, Milonia L, Tassinari J, Castagna A, Brandi C, Grimaldi L, D’Aniello C, Nisi G. Complications associated with brachioplasty: a literature review. Acta Biomed 2018;88:393-402.

23. Boccara D, Petit A, Reinbold C, Chaouat M, Mimoun M, Serror K. The M-Y axilloplasty after massive weight loss: analysis of 159 consecutive patients. Aesth Plast Surg 2018;117:403.

24. Hoyos A, Perez ME, Guarin DE, Montenegro A. A report of 736 high definition lipoabdominoplasties performed in conjunction with circumferential Vaser liposuction. Plast Reconstr Surg 2018; doi: 10.1097/PRS.0000000000004705.

25. Vico PG, Vooght A de, Nokerman B. Circumferential body contouring in bariatric and non-bariatric patient. J Plast Reconstr Aesthet Surg 2010;63:814-9.

26. Correia-Gonçalves I, Valença-Filipe R, Carvalho J, Rebelo M, Peres H, Amarante J, Costa-Ferreira A. Abdominoplasty with Scarpa fascia preservation - comparative study in a bariatric population. Surg Obes Relat Dis 2017;13:423-8.

27. Ardehali B, Fiorentino F. A meta-analysis of the effects of abdominoplasty modifications on the incidence of postoperative seroma. Aesthet Surg J 2017;37:1136-43.

28. Schmidt M, Pollhammer MS, Januszyk M, Duscher D, Huemer GM. Concomitant liposuction reduces complications of vertical medial thigh lift in massive weight loss patients. Plast Reconstr Surg 2016;137:1748-57. 